\title{
SIMULTANEOUS MULTI-FREQUENCY IMAGING OF THE NUCLEUS OF NGC 1275
}

\author{
J.D. ROMNEY ${ }^{1}$, R.C. WALKER ${ }^{1}$, K.I. KELLERMANN ${ }^{2}$, \\ R.C. VERMEULEN ${ }^{3}$ AND V. DHAWAN ${ }^{1}$ \\ ${ }^{1}$ NRAO - Socorro, NM, USA \\ ${ }^{2}$ NRAO - Charlottesville, VA, USA \\ ${ }^{3}$ Caltech - Pasadena, CA, USA
}

\section{Introduction}

An unusual counterjet feature was discovered in $3 \mathrm{C} \mathrm{84,} \mathrm{the} \mathrm{compact} \mathrm{radio}$ nucleus of NGC 1275, in the "First Science" observations on the VLBA at 8.4 GHz (Walker et al., 1994), and simultaneously in Global VLBI observations at $22 \mathrm{GHz}$ (Vermeulen et al., 1994). Comparison of these images indicated a strongly inverted spectrum in this feature, but the interpretation was clouded by the two-year difference between the epochs of observation. To resolve this ambiguity, and to study the spectrum of the counterjet, we exploited the capabilities of the VLBA to make nearly simultaneous observations of $3 \mathrm{C} 84$ at $2.3,5.0,8.4,15.4,22$, and $43 \mathrm{GHz}$, in four apparitions over a 16-day period in January 1995. These observations also served to continue structural monitoring programs at 15 and $22 \mathrm{GHz}$. This paper presents preliminary images from those observations. A companion contribution by Walker et al. (1995) discusses the interpretation of the images.

\section{Observations}

Individual observing sessions were scheduled at $15.4,22$, and $43 \mathrm{GHz}$, while simultaneous observations at 2.3 and $8.4 \mathrm{GHz}$ alternated with $5.0 \mathrm{GHz}$ in a fourth session. All ten VLBA stations participated, except that high winds disabled the Mauna Kea station in the $15 \mathrm{GHz}$ session, and an instrumental failure occurred at Fort Davis in the multi-frequency session. The observa- 

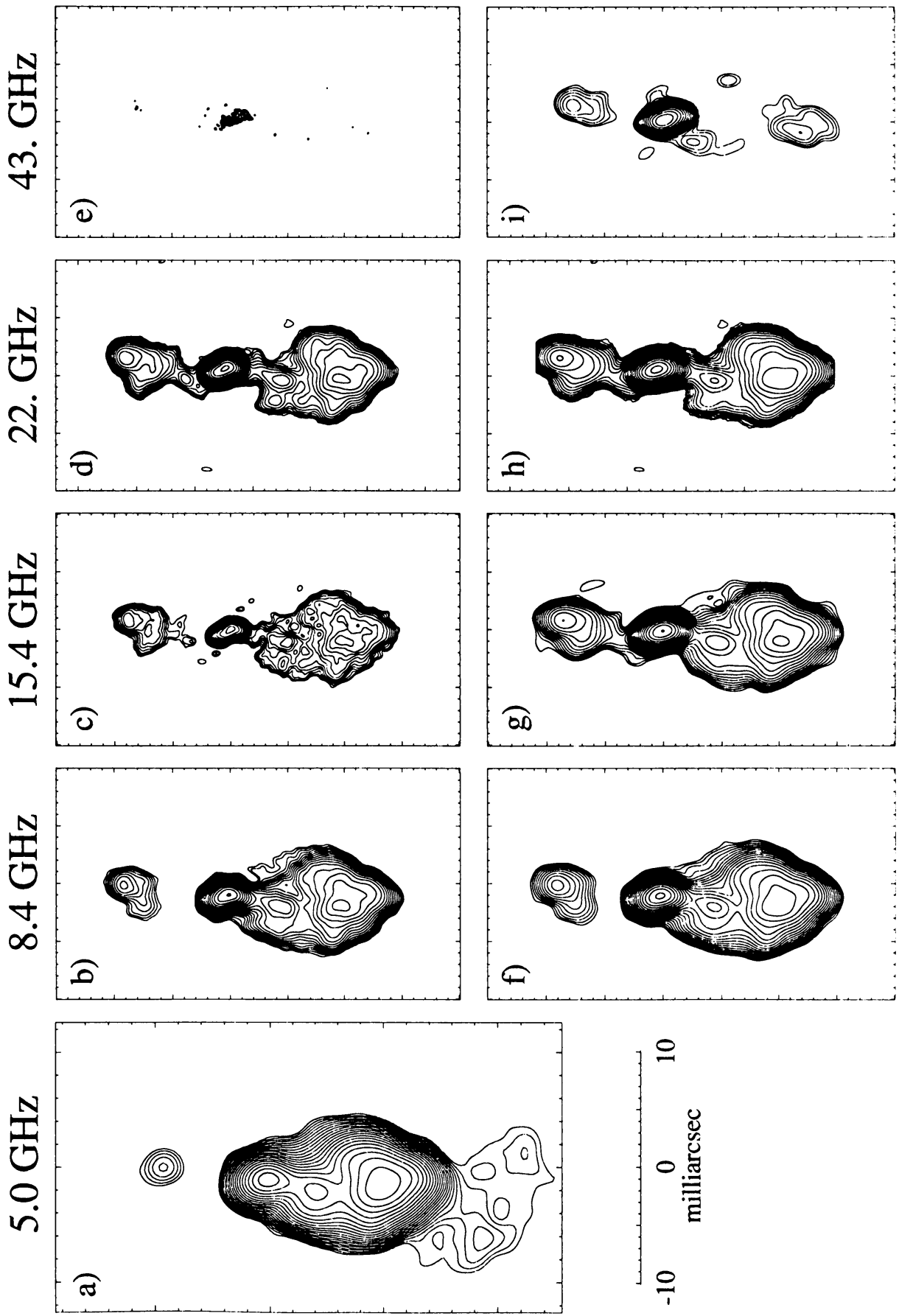

Figure 1. Images of $3 \mathrm{C} 84$ at five frequencies. The image pairs at the highest four frequencies are explained in the text. The common angular scale is shown in the lower left. A common set of contour levels are used, with the lowest contour at $5 \mathrm{mJy} /$ beam except in (a) $1.86-\mathrm{mJy} /$ beam and (e) $13.3 \mathrm{mJy} / \mathrm{beam}$. Ascending contours are logarithmic with seven steps per decade $(1 ., 1.39,1.93,2.68,3.73,5.18,7.20,10 ., \ldots)$. 

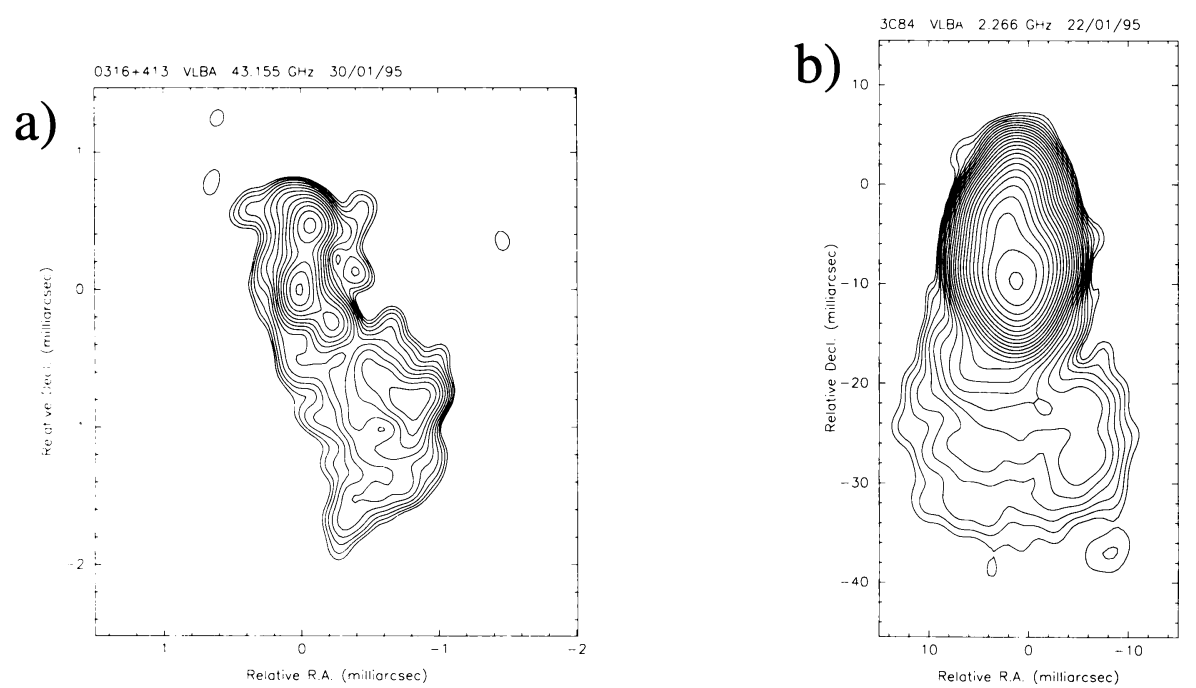

Figure 2. (a) Fine-scale image at $43 \mathrm{GHz}$. (b) Extended image at $2.3 \mathrm{GHz}$. The lowest contour in both images is $2.5 \mathrm{mJy} / \mathrm{beam}$, with the same logarithmic ascending contours used in Figure 1.

tions were correlated within 4 weeks, and imaged using primarily the AIPS system, as well as the DIFMAP and VISAMAT programs.

Figure 1 presents two different views of the images at the five highest frequencies. The upper set of images have been restored with the conventional approximation to the synthesized beam. Those in the lower row have been restored with the beam appropriate to the $5.0-\mathrm{GHz}$ observations, to facilitate comparisons and to bring out the jet and counterjet structures at $43 \mathrm{GHz}$. The $43-\mathrm{GHz}$ image also required a substantial $(u, v)$ taper in the imaging process to reveal the counterjet. The images are aligned relative to the central "core" component which is brightest at most frequencies.

Two images which can not be conformed to this scheme are shown separately. Figure 2a presents the core region at $43 \mathrm{GHz}$, at a scale more suitable to the extremely compact structures which exist at that frequency. Figure $2 \mathrm{~b}$ is the lowest-frequency image, at $2.3 \mathrm{GHz}$, which is sufficiently extended that it could not be combined with those at the higher frequencies.

We gratefully acknowledge the contributions of W. Alef, D. C. Backer, J. M. Benson, and A. C. S. Readhead to the 3C 84 observational program of which this paper is part.

\section{References}

Vermeulen, R.C., Readhead, A.C.S., and Backer, D.C. (1994), Ap. J., 430, L41.

Walker, R.C., Romney, J.D., and Benson, J.M. (1994) Ap. J., 430, L45.

Walker, R.C. et al. (1995) This Volume. 